\title{
Acute Necrotizing Pancreatitis and Coronavirus Disease-2019 (COVID-19)
}

\author{
Ulaş Aday, Ercan Gedik ${ }^{1}$, Mehmet Tolga Kafadar ${ }^{1}$ and Erdal Özbek ${ }^{2}$ \\ Departments of Gastrointestinal Surgery, Surgery ${ }^{1}$, and Clinical Microbiology ${ }^{2}$, Dicle University School of Medicine, Diyarbakır, Turkey
}

Coronavirus disease-19 (COVID-19) is caused by the severe acute respiratory syndrome coronavirus-2 (SARS-CoV-2) and has resulted in increased mortality worldwide. Several studies have identified the involvement of the gastrointestinal tract, respiratory tract, and other tissues. Although it has been reported that the angiotensin-converting enzyme-2 receptor affected by SARS-CoV is expressed more in the pancreas than in the lungs, the issue regarding the occurrence of pancreatitis is controversial. SARS Cov- 2 rarely causes acute necrotizing pancreatitis without significantly affecting the respiratory and other systems. This paper presents a patient who underwent laparotomy due to acute necrotizing pancreatitis and hemodynamic instability caused by COVID-19 without any known risk factors. (Korean J Gastroenterol 2021;78:353-358)

Key Words: COVID-19; Pancreatitis, acute necrotizing; SARS-CoV-2

\section{INTRODUCTION}

An increasing number of confirmed cases and deaths are caused by the severe acute respiratory syndrome coronavirus 2 (SARS-CoV-2) contributing to the coronavirus disease 2019 (COVID-19) pandemic. Coronaviruses are a large family of single-stranded RNA viruses, with infectivity in humans and animals, causing a myriad of symptoms. ${ }^{1}$ Acute pancreatitis is often caused by gallstones and alcohol abuse, rarely of a viral origin. The most common causes of viral pancreatitis are hepatitis viruses (A, B, C, D, and E), coxsackie, hemorrhagic fever viruses, cytomegalovirus, varicella zoster, mumps, and measles. ${ }^{2}$

Data on the frequency and clinical course of acute pancreatitis caused by SARS-CoV-2 are controversial, but it is believed to increase multi-organ failure (MOF), morbidity, and mortality. ${ }^{3,4}$ Angiotensin-converting enzyme-2 (ACE2), the functional virus-host cell receptor, is expressed in both exocrine and endocrine pancreatic cells and plays a role in this disease process. Pancreatic damage can occur through various mechanisms, including the direct cytopathic effects of SARS-CoV-2 and indirect systemic inflammatory and immune-mediated cellular responses that cause organ damage.,6 This paper presents a COVID-19 case who presented with severe acute necrotizing pancreatitis (ANP) and underwent a laparotomy without any known risk factors.

\section{CASE REPORT}

A 32-year-old male patient presented to the emergency department at another healthcare center with sudden-onset abdominal pain and nausea. In that center, the patient was hospitalized with a diagnosis of acute pancreatitis after showing a lipase level of 1,236 (normal range 0-60) U/L and an amylase level of 738 (normal range 28-100) U/L. Abdominal ultrasonography (USG) showed no abnormality related to the gall-

Received September 30, 2021. Revised October 20, 2021. Accepted October 20, 2021.

(C) This is an open access article distributed under the terms of the Creative Commons Attribution Non-Commercial License (http://creativecommons.org/licenses/ by-nc/4.0) which permits unrestricted non-commercial use, distribution, and reproduction in any medium, provided the original work is properly cited. Copyright (C) 2021. Korean Society of Gastroenterology.

Correspondence to: Ulaş Aday, Department of Gastrointestinal Surgery, Dicle University School of Medicine, Yukarıkılıçtaşı Mh., 21200 Sur/Diyarbakır, Turkey. Tel: +90-412-248-80 01-4777, Fax: +90-412-248-8216, E-mail: ulasaday@gmail.com, ORCID: https://orcid.org/0000-0002-3161-0923

Financial support: None. Conflict of interest: None. 
bladder and bile ducts, while unenhanced abdominopelvic CT showed heterogeneity in the pancreatic head and body parenchyma, increased density in the peripancreatic fatty mesentery, and minimal peripancreatic and periduodenal fluid, all of which were consistent with pancreatitis (Fig. 1). At the 12th-hour follow-up, the patient underwent emergency surgery due to the development of hemodynamic instability, acute abdominal findings (rebound, defense), and shock. A laparotomy was performed after detecting retroperitoneal hemorrhage in the laparoscopic examination. Approximately $500 \mathrm{~mL}$ of necrotic-hemorrhagic intraabdominal fluid was drained, and the procedure was completed after packing. Perioperatively, the patient received two units of erythrocyte suspension and two units of fresh frozen plasma. After surgery, the patient was transferred to the Dicle University School of Medicine, Gastrointestinal Surgery Clinic and transported to the intensive care unit intubated. Upon admission, his heart rate and arterial blood pressure were 115 beats/min and 135/72 mmHg, respectively, and he was oliguric. The laboratory parameters suggested pancreatitis (Table 1). Supportive treatment and meropenem ther- apy was initiated. The detailed medical history indicated no known history of congenital/chronic diseases, trauma, or alcohol and drug abuse. His wife was diagnosed with COVID-19 2 weeks earlier and was in home isolation, while the patient had a negative swab test one week earlier. Accordingly, a RT-PCR assay was performed, and he was isolated after testing positive on the test. Abdominal CT angiography was performed to evaluate a possible intraabdominal hemorrhage and plan the endovascular intervention. Although it showed no findings consistent with contrast media extravasation or bleeding, the results were suggestive of ANP, and compresses were placed in the previous laparotomy (Fig. 1). Pleural effusion was observed in thoracic sections, while no findings consistent with COVID-19 were observed (Fig. 2). At the end of the first day of follow-up, his body temperature started to increase. Despite no signs of active bleeding, the patient was considered to have abdominal compartment syndrome because of the increased intraabdominal pressure and severe renal dysfunction. The intravesical pressure, which was measured every 6 hours in the first 48 hours after being admitted to the intensive
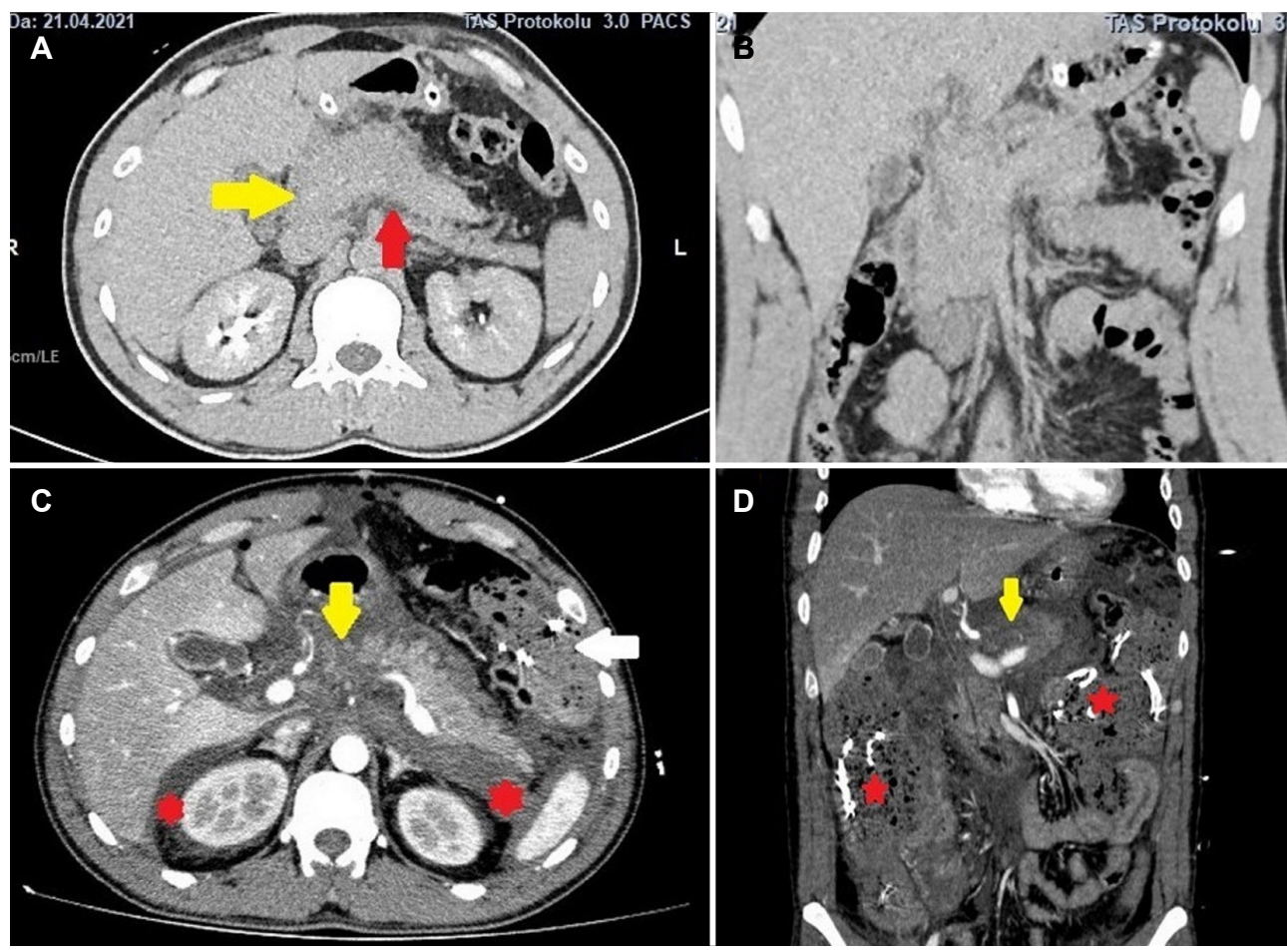

Fig. 1. Pancreatic edema (yellow arrow of A) and minimal peripancreatic fluid (red arrow of A) are observed in (A) axial and (B) coronal sections of the uncontrasted computed tomography (CT) taken after the initial diagnosis. These images were taken before laparotomy in the center where the first hospitalization was performed. (C, D) CT angiography taken in the Dicle University School of Medicine, Gastrointestinal Surgery Clinic revealed necrosis in the pancreas (yellow arrow), increased fluid extending to the perirenal area (red stars), and compresses placed in the previous laparotomy (white arrow of C, red stars of D). 
Table 1. Laboratory Parameters at the Time of Diagnosis, at the 48th Hour and on the 10th Day

\begin{tabular}{|c|c|c|c|c|}
\hline Parameter & Normal range & Time of first diagnosis & Forty-eight hours after diagnosis & 10th day \\
\hline White cell count & $3.9-8.7 \times 10^{9} / \mathrm{L}$ & 16.9 & 7.47 & 14.2 \\
\hline Total neutrophils & $1.8-7.4 \times 10^{9} / \mathrm{L}$ & 14.4 & 6.56 & 11 \\
\hline Total lymphocytes & $0.85-3 \times 10^{3} / \mathrm{uL}$ & 0.90 & 0.45 & 1.66 \\
\hline Platelet & $151-304 \times 10^{9} / \mathrm{L}$ & 146 & 138 & 531 \\
\hline Hemoglobin & $11.9-15.4 \mathrm{~g} / \mathrm{dL}$ & 7.8 & 9.4 & 8.1 \\
\hline Lipase & $0-60 \mathrm{U} / \mathrm{L}$ & 1,236 & 542 & 21 \\
\hline Amylase & $28-100 \mathrm{U} / \mathrm{L}$ & 738 & 473 & 138 \\
\hline Creatinine & $0.3-1.2 \mathrm{mg} / \mathrm{dL}$ & 1.01 & 2.09 & 0.8 \\
\hline Urea & $16.6-48.5 \mathrm{mg} / \mathrm{dL}$ & 53 & 79.7 & 41.8 \\
\hline Lactate dehydrogenase & $0-248 \mathrm{U} / \mathrm{L}$ & - & 1,585 & 863 \\
\hline Alanine aminotransferase & $0-40 \mathrm{U} / \mathrm{L}$ & 153 & 37.1 & 37.1 \\
\hline Aspartate aminotransferase & $0-40 \mathrm{U} / \mathrm{L}$ & 153 & 57.5 & 44.4 \\
\hline Total bilirubin & $0.3-1.2 \mathrm{mg} / \mathrm{dL}$ & 1.3 & 1.42 & 0.92 \\
\hline Alkaline phosphatase & $30-120 \mathrm{U} / \mathrm{L}$ & - & 56 & 296 \\
\hline Gamma-glutamyltransferase & $0-55 \mathrm{U} / \mathrm{L}$ & - & 87.5 & 644 \\
\hline C-reactive protein & $0.0-0.5 \mathrm{mg} / \mathrm{dL}$ & 18 & 17.5 & 3.15 \\
\hline Procalcitonin & $0-0.05 \mu \mathrm{g} / \mathrm{L}$ & - & 1 & 0.23 \\
\hline Triglyceride & $<200 \mathrm{mg} / \mathrm{dL}$ & - & 181 & - \\
\hline Calcium & $8.4-10.4 \mathrm{mg} / \mathrm{dL}$ & 7.39 & 6.81 & 7.2 \\
\hline D-dimer & $0-500 \mathrm{ng} / \mathrm{mL}$ & 1,479 & 950 & 8,450 \\
\hline Fibrinogen & $180-400 \mathrm{mg} / \mathrm{dL}$ & - & 659 & 445 \\
\hline Ferritin & $30-400 \mathrm{ng} / \mathrm{mL}$ & 841 & - & - \\
\hline
\end{tabular}
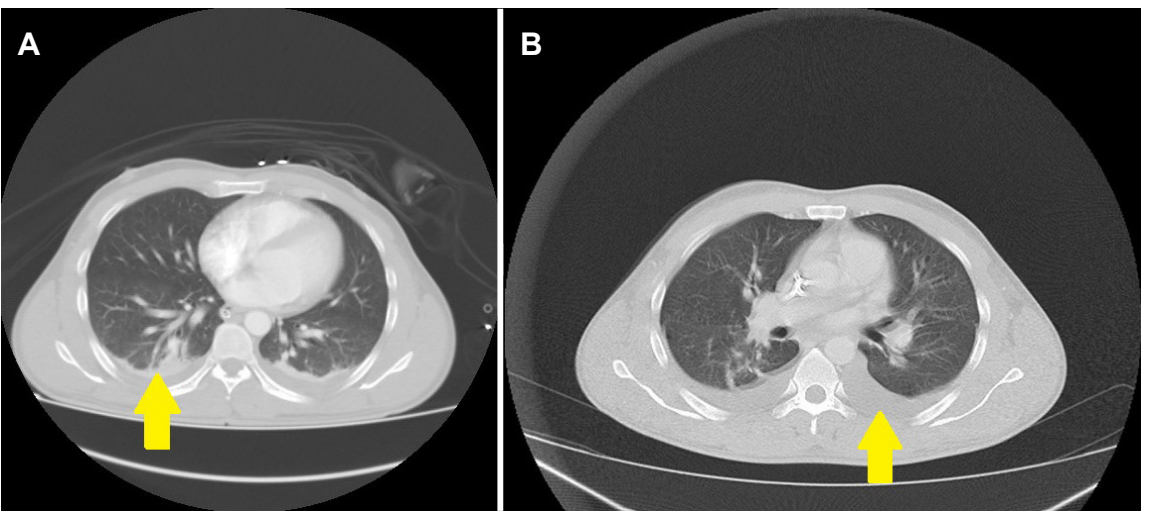

Fig. 2. (A, B) Pleural effusion in the thorax sections of the computed tomography taken at the first diagnosis and 10 days later, but no findings associated with coronavirus disease2019 were observed (arrows).
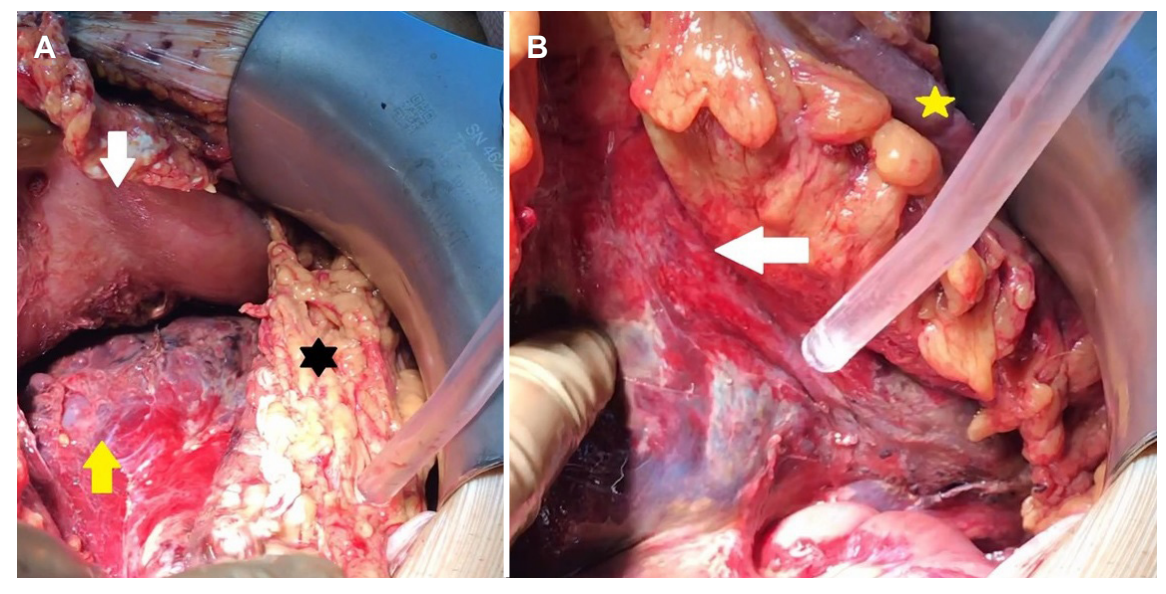

Fig. 3. Intraoperative view. (A) When the gastrocolic ligament was opened, and the stomach (white arrow) was pulled superiorly, necrosis and hemorrhage areas were observed in the pancreatic corpus (yellow arrow), lipolysis areas (black star) in the omentum, (B) hemorrhagic-necrotic penetration into the mesocolon (white arrow) was observed when the colon (yellow star) was suspended. 
care unit, was above $20 \mathrm{mmHg}$. The patient underwent a relaparotomy 48 hours after the first laparotomy to remove the compresses. An abdominal exploration showed no active bleeding while it revealed an impression consistent with ANP (Fig. 3). Necrotic abdominal fluid was sampled to analyze the presence of SARS-CoV-2. The abdominal cavity was irrigated with $3 \mathrm{~L}$ of saline. A drain was placed in the anterior pancreatic head and the rectovesical pouch. Only the skin was closed to reduce the intraabdominal pressure.

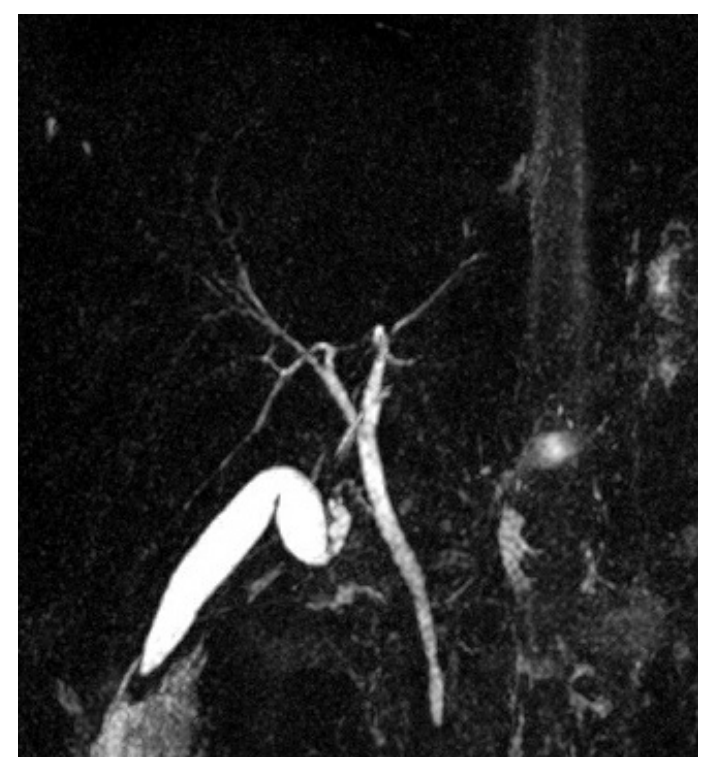

Fig. 4. Magnetic resonance pancreaticolangiography showing no extrahepatic-intrahepatic biliary duct dilatation and homogeneous contrast in the gallbladder, demonstrating no evidence of choledocholithiasis or cholelithiasis.
After surgery, the patient was taken to the COVID-19 clinic extubated. The abdominal fluid was positive for SARS-CoV-2 on RT-PCR. Parenteral nutrition was initiated because of insufficient postoperative oral intake. Octreotide was administered intravenously for the first 7 days $(3 \times 1)$ and then administered as a depot injection. No stones or sludge was detected in the etiology of pancreatitis on USG or magnetic resonance pancreaticolangiography (Fig. 4). The serum calcium level, immunoglobulin G4 level, lipid panel, and other viral tests were unremarkable. The pancreatitis was considered to be caused by SARS-CoV-2 because the patient had no known alcohol and drug abuse. A fat-free diet was initiated on postoperative day eight, and no pancreatic insufficiency developed because the patient showed good tolerance. On postoperative day 10 , repeat $C T$ showed significant regression in the pancreatic edema and necrosis (Fig. 5). The swab test was negative on day 16 , and no respiratory failure occurred throughout the clinical follow-up. The patient was discharged uneventfully on postoperative day 20.

The authors are accountable for all aspects of the work in ensuring that questions related to the accuracy or integrity of any part of the work are appropriately investigated and resolved. All procedures performed in studies involving human participants were in accordance with the ethical standards of the institution and with the Helsinki Declaration (as revised in 2013). Written informed consent was obtained from the patient.
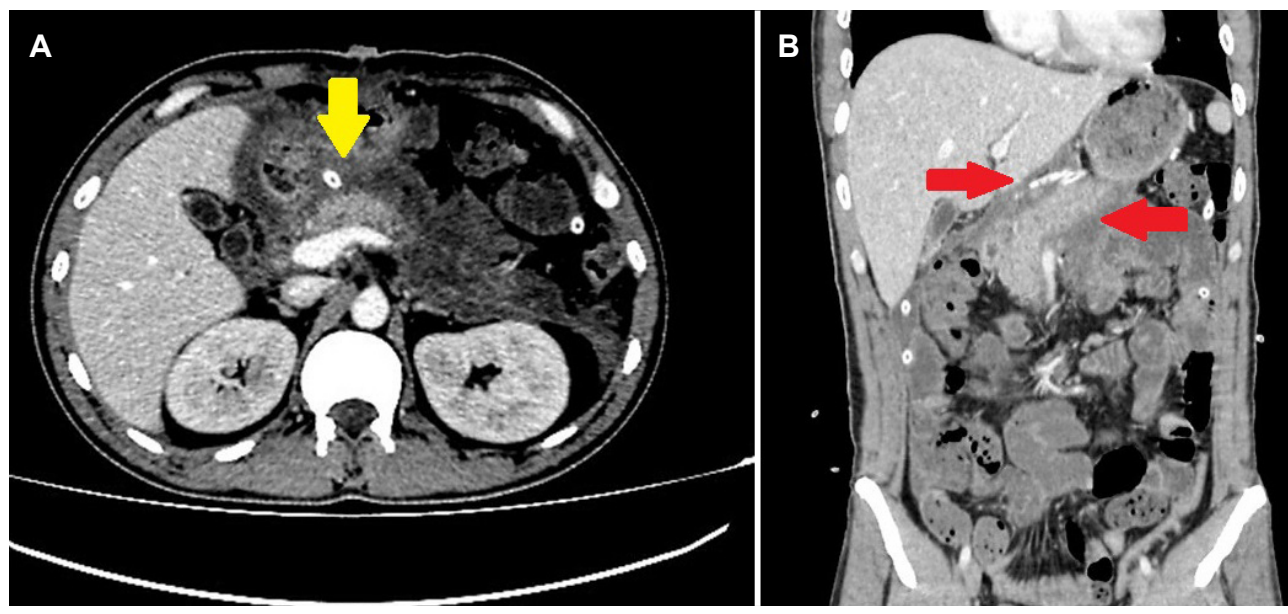

Fig. 5. (A) Contrast-enhanced computed tomography sections of the 10th day revealed regression of intra-abdominal fluids (yellow arrow) and (B) significant improvement in ischemic areas in the pancreatic parenchyma (red arrows). 


\section{DISCUSSION}

Acute pancreatitis is a common disease with many causes, but the etiology is unknown in $15-25 \%$ of cases. There is insufficient evidence that SARS-CoV-2 specifically causes pancreatitis. ${ }^{7}$ According to a retrospective cohort study analyzing 11,883 COVID-19 positive patients hospitalized in 12 centers in the USA, only 32 patients $(0.27 \%)$ had pancreatitis. ${ }^{8}$ A similar study conducted in Spain involving 63,000 COVID-19 patients reported a $0.007 \%$ frequency of acute pancreatitis. According to the results of this study, there is a low risk of acute pancreatitis. ${ }^{9}$

Several case reports and small studies have suggested a causal relationship between COVID-19 and acute pancreatitis. Necrosis and severe pancreatitis are seen in a very small number of cases. Lipase elevation and mild edematous pancreatitis on radiological evaluation were observed in most of them. ${ }^{3,4,10-12}$ Wang et al. $^{13}$ reported the presence of ACE2 receptors in pancreatic islets and exocrine cells and stated that pancreatitis could be caused by SARS-CoV-2. Although the presence of ACE2 receptors in the pancreas makes it plausible, there is little robust data. The ACE2 receptors in the pancreas express pancreatic triglyceride lipase (PNLIP). PNLIP activation results in adipose lipolysis leading to acute lung injury and multisystem organ failure. ${ }^{14}$ There are contrasting opinions regarding the cytopathic mechanism of action of COVID-19. Pancreatitis in patients hospitalized for COVID-19 who developed MOF has been attributed to impairment of microcirculation and hypoxia. ${ }^{15}$ Kumar et al. ${ }^{4}$ reported that COVID-19 patients with acute pancreatitis at admission had a better prognosis and overall better outcomes than patients who developed acute pancreatitis during hospitalization.

In this case, there are data supporting that SARS-CoV-2 causes ANP: 1) no cause was found in the detailed evaluation of the pancreatitis etiology; 2) the RT-PCR test was positive in nasopharyngeal swab and necrotic abdominal pancreatic fluid; 3) there was pancreatitis from the beginning in the radiological evaluation; 4) the classical ANP picture was confirmed in laparotomy. The virus may have affected the primary pancreas from the beginning. Because respiratory failure did not develop in the clinical follow-up, and the COVID-19 findings were not observed in the computed tomography of the thorax taken both at admission and 10 days later. Despite the diagnosis of acute pancreatitis, the laparotomy without adequate supportive treatment should be questioned in this patient. Laparotomy in pancreatitis should be performed with careful patient selection; otherwise, the morbidity and mortality will increase. Coexistence with COVID-19 will increase this risk further.

In conclusion, SARS-CoV-2 is a rare cause of ANP. Clinicians should consider COVID-19 when evaluating the causes of pancreatitis. This diagnosis is important for adequate treatment and better management of the systemic responses that reduce the SARS-CoV-2 mortality.

\section{REFERENCES}

1. Guan WJ, Ni ZY, Hu Y, et al. Clinical characteristics of coronavirus disease 2019 in China. N Engl J Med 2020;382:1708-1720.

2. Imam Z, Simons-Linares $C R$, Chahal P. Infectious causes of acute pancreatitis: a systematic review. Pancreatology 2020;20: 1312-1322.

3. Dirweesh A, Li Y, Trikudanathan G, Mallery JS, Freeman ML, Amateau SK. Clinical outcomes of acute pancreatitis in patients with coronavirus disease 2019. Gastroenterology 2020;159: 1972-1974.

4. Kumar V, Barkoudah E, Souza DAT, Jin DX, McNabb-Baltar J. Clinical course and outcome among patients with acute pancreatitis and COVID-19. Eur J Gastroenterol Hepatol 2021;33: 695-700.

5. Patel KP, Patel PA, Vunnam RR, et al. Gastrointestinal, hepatobiliary, and pancreatic manifestations of COVID-19. J Clin Virol 2020 Apr 29. [Epub ahead of print]

6. Liu F, Long X, Zhang B, Zhang W, Chen X, Zhang Z. ACE2 expression in pancreas may cause pancreatic damage after SARS-CoV-2 Infection. Clin Gastroenterol Hepatol 2020;18: 2128-2130.e2.

7. de-Madaria E, Capurso G. COVID-19 and acute pancreatitis: examining the causality. Nat Rev Gastroenterol Hepatol 2021; 18:3-4.

8. Inamdar S, Benias PC, Liu Y, et al. Prevalence, risk factors, and outcomes of hospitalized patients with coronavirus disease 2019 presenting as acute pancreatitis. Gastroenterology 2020;159:2226-2228.e2.

9. Miró Ò, Llorens P, Jiménez S, et al. Frequency of five unusual presentations in patients with COVID-19: results of the UMC-19-S1. Epidemiol Infect 2020;148:e189.

10. Kataria S, Sharif A, Ur Rehman A, Ahmed Z, Hanan A. COVID-19 Induced acute pancreatitis: a case report and literature review. Cureus 2020;12:e9169.

11. Mohammadi Arbati M, Molseghi MH. COVID-19 Presenting as acute necrotizing pancreatitis. J Investig Med High Impact Case Rep 2021;9:23247096211009393.

12. Alves AM, Yvamoto EY, Marzinotto MAN, Teixeira ACS, Carrilho FJ. SARS-CoV-2 leading to acute pancreatitis: an unusual presentation. Braz J Infect Dis 2020;24:561-564. 
13. Wang F, Wang H, Fan J, Zhang Y, Wang H, Zhao Q. Pancreatic injury patterns in patients with coronavirus disease 19 pneumonia. Gastroenterology 2020;159:367-370.

14. Hegyi P, Szakács Z, Sahin-Tóth M. Lipotoxicity and cytokine storm in severe acute pancreatitis and COVID-19. Gastroenterology
2020;159:824-827.

15. Bulthuis MC, Boxhoorn L, Beudel M, et al. Acute pancreatitis in COVID-19 patients: true risk? Scand J Gastroenterol 2021;56: 585-587. 